\section{Hedgehog battles Down syndrome}

\section{By Lev Osherovich, Senior Writer}

Researchers at The Johns Hopkins University School of Medicine have proposed that mental retardation caused by Down syndrome could be prevented or mitigated after birth with a small molecule activator of the sonic hedgehog homolog pathway. ${ }^{1}$ The group's mouse data show improvements in brain development, learning and memory. However, the narrow therapeutic window and the long gap in time between treatment and evaluation of cognitive endpoints in patients will pose significant translational challenges.

Down syndrome arises from an extra copy of chromosome 21 , leading to multiple craniofacial and neurological abnormalities. Compared with brains from healthy controls, the brains of patients with Down syndrome have smaller cerebellums and hippocampuses-regions that control motor activity and memory, respectively.

\section{"This study makes me} excited to pursue the effects of a smoothened agonist on the hippocampus."

-Roger Reeves,

The Johns Hopkins University School of Medicine more cellularized cerebellums than those in vehicle-treated controls. Indeed, the cerebellums of the treated mice resembled those of wild-type animals.

The team next examined the effect of the SMO agonist on hippocampal function. Mice receiving the compound had improved learning and memory and performed better in a widely used maze navigation test than untreated controls. In a brain slice assay of hippocampal function, tissue from treated mice was more responsive to stimulation than that of untreated controls.

Reeves' team did not report whether the SMO agonist affected hippocampal morphology.

Results were published in Science Translational Medicine and are not

\section{Stimulating development}

Reeves' findings are surprising because the hedgehog pathway was not previously thought to affect development of the hippocampus during the postnatal period. What remains unclear is whether the hedgehog pathway directly acts in the hippocampus.

Because the cerebellum was previously believed to be the principal site of hedgehog signaling, one possibility is that having a bigger cerebellum could lead to improvements in other areas of the brain, including the hippocampus.

"The cerebellum is involved in motor function but is not thought to affect learning and memory," said Reeves. "Perhaps we have fixed something in the cerebellum and this has

The likely cause of this small brain size is deficient signaling by the sonic hedgehog homolog ( $\mathrm{SHH}$ ) pathway, which controls the duration of stem and progenitor cell proliferation throughout development.

In 2006, a team led by Roger Reeves found that in a mouse model of Down syndrome, the neonatal brain did not respond properly to hedgehog signaling and had fewer mature neurons than controls. ${ }^{2}$

"People with Down syndrome have a smaller cerebellum [than healthy controls], and we found that the mice do as well," said Reeves, who is a professor of physiology at the Institute of Genetic Medicine at Johns Hopkins. "The trisomic mice had a layer of precursor neurons that had the same number as in control mice, but they don't divide as much" in response to hedgehog signaling.

In the new study, Reeves' team reported that the neuronal deficiency in the brains of young mice with Down syndrome could be corrected by transient treatment with a small molecule activator of smoothened (SMO), a GPCR that is a downstream effector of hedgehog signaling.

Drawing on earlier evidence from a University of California, San Francisco study that suggested pharmacological activation of hedgehog pathway signaling promoted the proliferation of cerebellar neural precursors, ${ }^{3}$ Reeves hypothesized that a hedgehog agonist could correct the shortage of cerebellar neurons in Down syndrome mice.

Reeves' team tested this hypothesis by injecting newborn Down syndrome mice with a small molecule SMO agonist and observed larger, effects elsewhere in the brain."

In support of this idea, David Rowitch, a Howard Hughes Medical Institute investigator and a professor of pediatrics and neurosurgery at UCSF, said that the new findings are in line with recent evidence that the cerebellum can influence development of other brain tissues.

In 2011, Rowitch's team reported that a SMO agonist could prevent damage to the cerebellum in neonatal mice exposed to glucocorticoids, which are used to treat respiratory problems in premature infants. ${ }^{3}$

"There's a lot of attention now to the cerebellum as being of primary importance in development of other brain areas," said Rowitch. "Further research is required to determine whether the effects of the hedgehog pathway on the cerebellum and hippocampus are related or distinct."

To resolve this question, Rowitch recommended genetic experiments to selectively activate hedgehog signaling in various brain regions.

Reeves favors the idea that hedgehog signaling might act directly on the hippocampus independently of its effects on the cerebellum.

Evidence to support this view comes from a second study in which Reeves collaborated with the laboratory of Chris De Zeeuw, a professor at the Netherlands Institute for Neuroscience, to test the effect of a SMO agonist on cerebellar function.

In that study, published this week in The Journal of Neuroscience, the team showed that a SMO agonist increased the size of the cerebellum. However, the molecule did not improve two specific functions of that 
tissue-motor learning and light adaption—that are compromised in Down syndrome mice. ${ }^{4}$

Thus, Reeves suspects that the greater size of the cerebellum in mice treated with a SMO agonist may be a red herring, and the truly important site of hedgehog signaling may be the hippocampus itself.

"This study makes me excited to pursue the effects of a smoothened agonist on the hippocampus," said Reeves.

\section{Clinical challenge}

Regardless of where hedgehog signaling acts in the brain, the functional benefits on learning and memory seen in mice make SMO activation an attractive therapeutic strategy for Down syndrome.

Reeves thinks that a therapy able to address even a subset of the neurological aspects of Down syndrome would help improve quality of life.

"I think this pathway certainly has therapeutic application for people born with Down syndrome," he said.

The biggest obstacles for the hedgehog activation strategy are concerns about the safety of turning on a developmental pathway that is involved in a range of cancers, including a common pediatric brain tumor-medulloblastoma.

Indeed, drug development around the hedgehog pathway has focused exclusively on inhibiting overactive SMO in cancer. The most advanced SMO antagonist is Erivedge vismodegib, marketed by Roche's Genentech Inc. unit to treat advanced basal cell carcinoma (BCC). It is in Phase II testing for medulloblastoma and other solid tumors. Curis Inc. co-developed Erivedge with Genentech.

"One has to be extremely careful about the potential toxicities and off-target effects" ofactivating hedgehog signaling, said Rowitch.

Nevertheless, Reeves and Rowitch noted that tumor growth likely requires chronic activation of the hedgehog pathway such as through gain-of-function mutations in SMO.

"The chances of transformation into a tumor are just theoretical because animal studies have not borne out a tumorigenic potential for a short course of treatment," said Rowitch. "The kinds of situations that lead to tumors are oncogenic mutations where the cells can never escape from elevated hedgehog signaling."

Reeves hopes to use clinical pathology data to narrow down the therapeutic window during which cerebellar and hippocampal neurons are most likely to respond to boosted hedgehog signaling.

He said that the ideal scenario would be that a brief course of a SMO agonist could be given over a relatively short period immediately after birth, when neurons are developing rapidly. However, it is not yet known how much stimulation would be needed for what length of time to elicit benefits without incurring cancer risk.
"It's clear this neurodevelopmental process in people starts at least at birth and goes on for several years," said Reeves. "Some studies suggest that this developmental process starts at or near birth, while other studies suggest a wave of proliferation occurs later in childhood."

Previous clinical trials in patients with Down syndrome have focused on improving cognitive functioning with neuromodulatory agents used for symptomatic treatment of age-related cognitive decline, which develops more rapidly in patients with Down syndrome than in healthy controls.

For example, Roche's RG1662, a negative allosteric modulator of $\mathrm{GABA}_{\mathrm{A}}$ receptor $\alpha_{5}$ (GABRA5), is in Phase I testing in adult patients with Down syndrome.

A neonatal prevention trial of the kind suggested by Reeves' work has never been attempted and thus faces technical, regulatory and possibly ethical challenges.

For example, because the cognitive effects of Down syndrome involve a combination of motor, speech and learning deficits that are difficult to distinguish until later in childhood, it is not clear how long a trial should be, when it should begin and what endpoints would be most appropriate.

Rowitch thinks the predictable and severe nature of Down syndrome makes a potentially disease-modifying interventional trial in infants an appealing idea, but he said companies might steer clear of the space.

"In neonates with Down syndrome, you have a very high likelihood of severe mental deficit, so parents can be desperate to do something," said Rowitch. "On the other hand, it's very risky to work in a population where adverse consequences of treatment could incur liabilities. Not many companies are willing to venture in neonatal therapeutics."

Osherovich, L. SciBX 6(37); doi:10.1038/scibx.2013.1017

Published online Sept. 26, 2013

\section{REFERENCES}

1. Das, I. et al. Sci. Transl. Med.; published online Sept. 4, 2013; doi:10.1126/scitranslmed.3005983

Contact: Roger H. Reeves, The Johns Hopkins University School of Medicine, Baltimore, Md.

e-mail: rreeves@jhmi.edu

2. Roper, R.J. et al. Proc. Natl. Acad. Sci. USA 103, 1452-1456 (2006)

3. Heine, V.M. et al. Sci. Transl. Med. 3, 105ra104 (2011)

4. Gutierrez-Castellanos, N. et al. J. Neurosci. 33, 15408-15413 (2013)

COMPANIES AND INSTITUTIONS MENTIONED

Curis Inc. (NASDAQ:CRIS), Lexington, Mass.

Genentech Inc., South San Francisco, Calif.

Howard Hughes Medical Institute, Chevy Chase, Md.

The Johns Hopkins University School of Medicine, Baltimore, Md.

Netherlands Institute for Neuroscience, Amsterdam, the

Netherlands

Roche (SIX:ROG; OTCQX:RHHBY), Basel, Switzerland

University of California, San Francisco, Calif. 